\title{
Continuous Quantum Phase Transition between an Antiferromagnet and a Valence-Bond Solid in Two Dimensions: Evidence for Logarithmic Corrections to Scaling
}

\author{
Anders W. Sandvik \\ Department of Physics, Boston University, 590 Commonwealth Avenue, Boston, Massachusetts 02215, USA
}

(Received 25 January 2010; published 26 April 2010)

\begin{abstract}
The antiferromagnetic to valence-bond-solid phase transition in the two-dimensional $J$ - $Q$ model (an $S=1 / 2$ Heisenberg model with four-spin interactions) is studied using large-scale quantum Monte Carlo simulations. The results support a continuous transition of the ground state, in agreement with the theory of "deconfined" quantum criticality. There are, however, large corrections to scaling, of logarithmic or very slowly decaying power-law form, which had not been anticipated. This suggests that either the $\mathrm{SU}(N)$ symmetric noncompact $C P^{N-1}$ field theory for deconfined quantum criticality has to be revised or that the theory for $N=2$ (as in the system studied here) differs significantly from $N \rightarrow \infty$ (where the field theory is analytically tractable).
\end{abstract}

DOI: 10.1103/PhysRevLett.104.177201

Valence-bond solid (VBS) states of two-dimensional (2D) quantum spin systems have been studied for more than two decades [1] and have recently come into renewed focus with the theory of "deconfined" quantum criticality (DQC) [2,3], which describes the transition between an antiferromagnetic (AFM) and a VBS ground state in terms of deconfinement of spinons. In addition to the interest in such AFM-VBS transitions in condensed-matter physics, there are also intriguing connections to deconfinement in gauge theories in particle physics [4]. To test the validity of the DQC scenario, and to obtain quantitative results for, e.g., predicted unusual critical exponents, unbiased numerical studies of quantum spin Hamiltonians with AFM-VBS transitions are necessary.

The " $J-Q$ " model was introduced recently [5] as an $\mathrm{SU}(2)$ symmetric spin system realizing the 2D AFM-VBS transition, following earlier work on related U(1) symmetric models [6,7]. It combines the standard Heisenberg antiferromagnet with four-spin interactions, which lead to local correlated bond singlets (valence bonds) and reduce the amplitudes of the longer valence bonds required [8] in an AFM state. The $J-Q$ model is free from "sign problems" [9], which prohibit quantum Monte Carlo (QMC) studies of frustrated spin systems such as the $J_{1}-J_{2}$ Heisenberg model [10], on which much of the past computational (exact diagonalization) research on VBS sates was focused. While series expansions [11] around various candidate states can give some insights, QMC methods [12], when applicable, are the only unbiased tools for studying 2D quantum phase transitions (in contrast to one dimension, where the density-matrix-renormalization-group method [13] is applicable) [14]. Being sign problem free, the $J-Q$ model (and generalizations of it [15]) have opened up new avenues for exploring magnetically quantum-disordered states and quantum phase transitions.

In this Letter, a large-scale, high-precision QMC study of the AFM-VBS transition in the $J-Q$ model is presented
PACS numbers: 75.10.Jm, 64.70.Tg, 75.40.Cx, 75.40.Mg

in order to further test the he DQC theory, and to settle discrepancies between previous studies $[5,16,17]$. The main point of contention is the order of the transition. In the DQC theory, it was argued that AFM-VBS transitions are generically continuous [2] and that the critical point for $\mathrm{SU}(N)$ spins corresponds to a noncompact (NC) $C P^{N-1}$ field theory [3]. This is at odds with the long-standing Landau-Ginzburg paradigm, where a direct transition between two states breaking unrelated symmetries should be first order (except at fine-tuned multicritical points). Ground state $[5,15]$ and finite-temperature [16] QMC studies of the $J-Q$ model show scaling behavior in good agreement with the DQC theory, including a dynamic exponent $z=1$, a rather large anomalous dimension $\eta_{\text {spin }} \approx 0.35$, and an emergent $\mathrm{U}(1)$ symmetry in the VBS phase (which in the theory is associated with spinon deconfinement). On the other hand, a QMC finite-size analysis by Jiang et al. would, if correct, require a firstorder transition [17]. A weakly first-order AFM-VBS scenario has been elaborated by Kuklov et al. [18,19], based on results for a lattice model claimed to realize the $\mathrm{NCCP}^{1}$ action, but other studies of the action have reached different conclusions [3].

Here it will be shown that the claimed first-order signals in the study by Jiang et al. [17] can be attributed to overinterpretations of QMC data affected by significant systematical and statistical errors. The results to be presented below were obtained with the stochastic series expansion (SSE) method [20,21], which is a finite-temperature QMC method free from systematical errors. There are no indications of a first-order transition, even in systems of spacetime volume 20 times larger than in [17]. However, the data are now of high enough quality to detect logarithmically weak deviations from the scaling forms expected at a $z=1$ critical point. Logarithmic corrections are well known consequences of marginal operators at criticality, which, although they have not been predicted theoretically in this case (in large- $N$ treatments of the $\mathrm{NCCP}^{N-1}$ theories 
$[2,22,23])$, cannot a priori be ruled out for $N=2$. A firstorder transition would lead to much more dramatic deviations from $z=1$.

Turning now to a quantitative discussion of the calculations, the $J-Q$ Hamiltonian [5] can be written as

$$
H=-J \sum_{\langle i j\rangle} C_{i j}-Q \sum_{\langle i j k l\rangle} C_{i j} C_{k l},
$$

where $C_{i j}$ is a bond-singlet projector for $S=1 / 2$ spins; $C_{i j}=1 / 4-\mathbf{S}_{i} \cdot \mathbf{S}_{j}$. In the $J$ term $i j$ are nearest neighbors on the square lattice, while $i j$ and $k l$ in the $Q$ term are on opposite edges of a $2 \times 2$ plaquette. Lattices of $N=L^{2}$ spins with periodic boundaries are used. Assuming $z=1$ (based on previous work $[5,16]$ ), the inverse temperature $\beta=Q / T$ is taken proportional to $L$ for finite-size scaling; $\beta=L$ and $\beta=L / 4$ will be considered for $L$ up to 256 . Calculations for $T / Q \geq 0.035$ are also carried out for systems sufficiently large, up to $L=512$, to give results in the thermodynamic limit.

The focus here will be on magnetic properties. The staggered magnetization $m_{s}$ is computed along the $z$ (quantization) axis. To extract the critical coupling ratio $(J / Q)_{c}$, and to address the issue of a possible first-order transition, consider first the Binder cumulant [24],

$$
U_{2}=\frac{5}{2}\left(1-\frac{1}{3} \frac{\left\langle m_{s z}^{4}\right\rangle}{\left\langle m_{s z}^{2}\right\rangle^{2}}\right)
$$

which is defined so that $U_{2} \rightarrow 0$ and $U_{2} \rightarrow 1$ in an AFM disordered and ordered state, respectively, when $L \rightarrow \infty$ (stemming from a Gaussian distribution of $\left|\vec{m}_{s}\right|$ around $\left|\vec{m}_{s}\right|=0$ and a $\delta$ function at $\left|\vec{m}_{s}\right|>0$, respectively). The factors in (2) correspond to $m_{s z}$ being one component of a three-dimensional vector $\vec{m}_{s}$. At a continuous transition, curves of $U_{2}$ versus $J / Q$ for different system sizes should intersect at the critical coupling, where normally $0<U_{2}<$ 1 [24]. At a first-order transition, on the other hand, $U_{2} \rightarrow$ $-\infty$ when $L \rightarrow \infty$ [24], following from a distribution with peaks at both $\left|\vec{m}_{s}\right|>0$ and $\left|\vec{m}_{s}\right|=0$ when the ordered and disordered phases coexist (with weight transferring rapidly

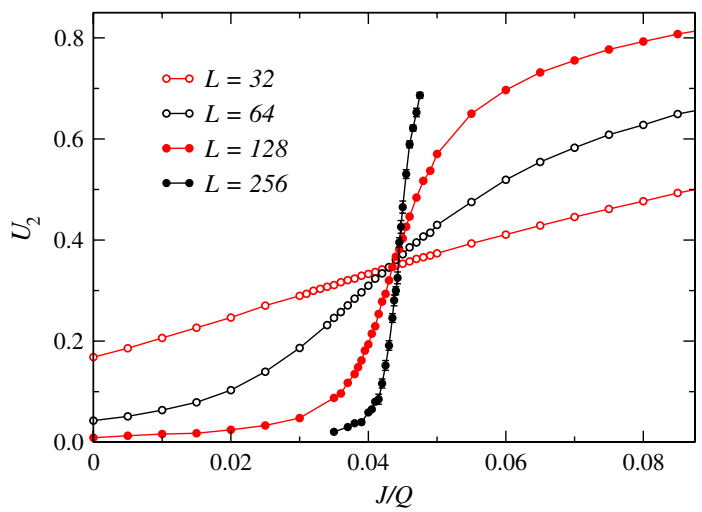

FIG. 1 (color online). Binder cumulant for the sublattice magnetization as function of the coupling ratio for different system sizes at inverse temperature $\beta=L$. between the peaks as the transition is crossed for large finite $L$ ). It should be noted that $U_{2}$ can be negative also at a continuous transition $[24,25]$ —only a divergence signals a first-order transition.

As seen in Fig. 1, in the $J-Q$ model there are no signs of $U_{2}$ becoming negative. The curves intersect at a point which moves very slowly toward larger $J / Q$ with increasing system size. The critical coupling for $L \rightarrow \infty$ can be extracted by extrapolating the crossing points for systems of size $L$ and $L / 2$, as shown in Fig. 2.

Figure 2 also shows results for the size-dependent critical coupling suggested by Kuklov et al. [18] and used by Jiang et al. [17]. It is based on the winding numbers,

$$
W_{a}=\frac{1}{L} \sum_{p=1}^{n} J_{a}(p)
$$

where $J_{a}(p), a=x, y$, is the spin current in lattice direction $a$ at location $p$ in a SSE configuration containing $n$ operators [20]. In the case of the $J-Q$ model, these currents take the values $J_{a}(p) \in\{0, \pm 1, \pm 2\}$. The "temporal" winding number is essentially the magnetization;

$$
W_{\tau}=2 M_{z}, \quad M_{z}=\sum_{i=1}^{N} S_{i}^{z} .
$$

The squared winding numbers are related to two important thermodynamic quantities: the spin stiffness,

$$
\rho_{s}=\frac{1}{2 \beta}\left(\left\langle W_{x}^{2}\right\rangle+\left\langle W_{y}^{2}\right\rangle\right),
$$

and the uniform magnetic susceptibility,

$$
\chi=\frac{\beta}{N}\left\langle M_{z}^{2}\right\rangle=\frac{\beta}{4 N}\left\langle W_{\tau}^{2}\right\rangle .
$$

For $L \rightarrow \infty$ and $T \rightarrow 0$, in a magnetically disordered (here VBS) phase $\rho_{s} \rightarrow 0$ and $\chi \rightarrow 0$, while in the AFM phase $\rho_{s}>0$ and $\chi>0$. A possible definition of the transition point (for finite $L$ and $\beta$ ) is the coupling at which the probability $P_{0}$ of all the winding numbers being zero is $1 / 2$ (or any fixed fraction) [18]. Figure 2 shows results obtained by interpolating $P_{0}$ for several $J / Q$ values. They extrapolate to the same $(J / Q)_{c} \approx 0.0445$ as the Binder

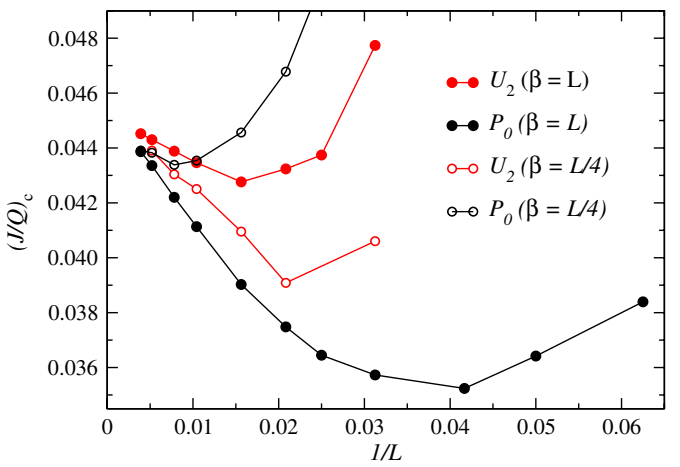

FIG. 2 (color online). Critical couplings extracted from the crossing of $U_{2}(L)$ and $U_{2}(L / 2)$ and from the winding number criterion $P_{0}=1 / 2$ in systems with $\beta=L$ and $L / 4$. 
cumulant crossings, but the corrections are larger. Note also that the size dependence varies significantly with the aspect ratio $\beta / L$. The results do not agree well with those of Jiang et al. [17], although $\rho_{s}$ and $\chi$ agree reasonably well for the system sizes available for comparisons. It is possible that $P_{0}$ is more sensitive to the Trotter approximation used in [17]. Note also the nonmonotonic size dependence in Fig. 2. An $\ln (L) / L^{3}$ convergence of $(\mathrm{J} / Q)_{c}$ was cited in [17] as a sign of a first-order transition. The data fits were, however, based on only three system sizes. The behavior for larger lattices is clearly different.

The $L \rightarrow \infty$ critical value $(J / Q)_{c} \approx 0.044$ is marginally higher than in previous studies. In particular, fitting the expected $z=1$ form $\chi \sim T$ of the susceptibility at $T>0$ $(L \rightarrow \infty)$, Melko and Kaul found $(J / Q)_{c} \approx 0.038$ [16]. At higher $J / Q$ they found $\chi=a+b T$, as expected in the AFM phase. Figure 3 shows $\chi / T$ down to temperatures less than half of the lowest $T$ considered in [16]. At $J / Q=$ 0.04 , while $\chi / T$ is roughly $T$ independent for $0.05 \lessgtr$ $T / Q \lesssim 0.2$, there is a drop at lower $T$, consistent with a spin-gapped phase. Close to the critical point there is no pure $\chi \propto T$ dependence at low $T$; instead the data exhibit a slow divergence, $\chi / T \approx a+b \ln (Q / T)$. The fanning out of the data suggests that the logarithmic form is a critical separatrix between the expected $T \rightarrow 0$ behaviors in the VBS and AFM phases.

Another indication of logarithmic corrections comes from the total squared winding number,

$$
\left\langle W^{2}\right\rangle=\left\langle W_{x}^{2}\right\rangle+\left\langle W_{y}^{2}\right\rangle+\left\langle W_{\tau}^{2}\right\rangle=2 \beta \rho_{s}+\frac{4 N}{\beta} \chi,
$$

for which Jiang et al. claimed an asymptotic linear divergence at the transition [17], as would be expected when AFM and VBS phases coexist at a first-order transition. Figure 4 shows the results of the present study. While $\left\langle W^{2}\right\rangle$ indeed grows with $L$, it does so very slowly, consistent with a logarithmic divergence. There is no plateau followed by a linear divergence - that conclusion [17] seems to be based on an overinterpretation of noisy data.

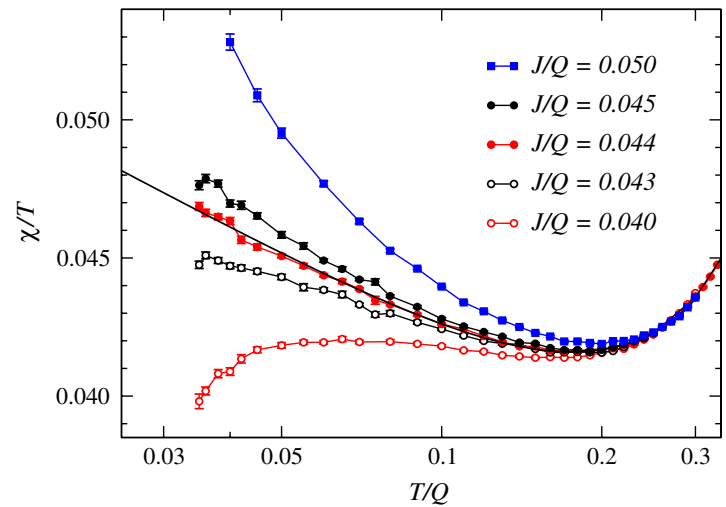

FIG. 3 (color online). The uniform susceptibility divided by the temperature in the neighborhood of the critical point. The solid curve is of the form $\chi / T=a+b \ln (Q / T)+c T^{2}$.
In principle, it is not possible to distinguish between a logarithm and a conventional scaling correction $\sim L^{-\omega}$ with a very small $\omega>0$. Figure 4 shows fits with $\omega=$ 0.1 along with the logarithmic form-when $\omega \rightarrow 0$ the two forms coincide exactly. This comparison shows that if the corrections are conventional, then $\omega \lesssim 0.1$. This is true also for the uniform susceptibility (Fig. 3).

Consider now the stiffness [not combined with $\chi$ as in (7)]. At a conventional $z=1$ critical point $\rho_{s} \sim 1 / L$. In the present case the drift in crossing points of $\rho_{S} L$ curves for different $L$ is larger than what is normally [26] expected, but can be compensated by a logarithm, $\rho_{s} L / \ln \left(L / L_{0}\right)$, as shown in Fig. 5. For $L \geq 48$ the curves intersect at a point, giving $(\mathrm{J} / Q)_{c}=0.0447 \pm 0.002$, in agreement with all the other results discussed above. Scaling fits away from the critical point give a correlation length exponent $\nu \approx 0.6$, but this is without considering possible corrections also to the conventional $L^{1 / \nu}$ scaling. It is difficult to include logarithmic corrections in quantities where the leading exponent is not known, in contrast to $\rho_{s}$ and $\chi$ where $z=1$ governs the leading behavior.

The conclusion of this study is that the AFM-VBS transition in the $J-Q$ model is continuous, but with significant corrections to the $z=1$ scaling that have not been discussed previously. The corrections appear to be logarithmic, although conventional scaling corrections $\sim L^{-\omega}$ with $\omega<0.1$ cannot be ruled out based on the numerical data alone. Regarding the possibility of a very weakly firstorder transition, it should be noted that rigorous proofs of continuous phase transitions are only available for a small number of exactly solvable models, yet accumulated numerical evidence of scaling (and experiments on natural

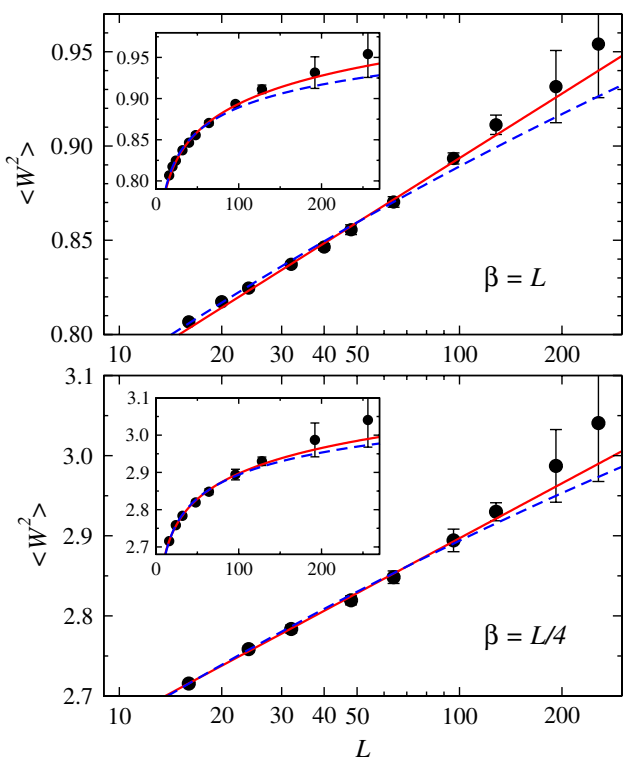

FIG. 4 (color online). Size dependence of the total winding number at the $P_{0}(L)=1 / 2$ point for $\beta / L=1$ and $1 / 4$. The data are shown on log-lin (main panels) and lin-lin scales (insets). The solid and dashed curves are fits to forms $a+b \ln (L)$ and $c-d L^{-0.1}$, respectively. 


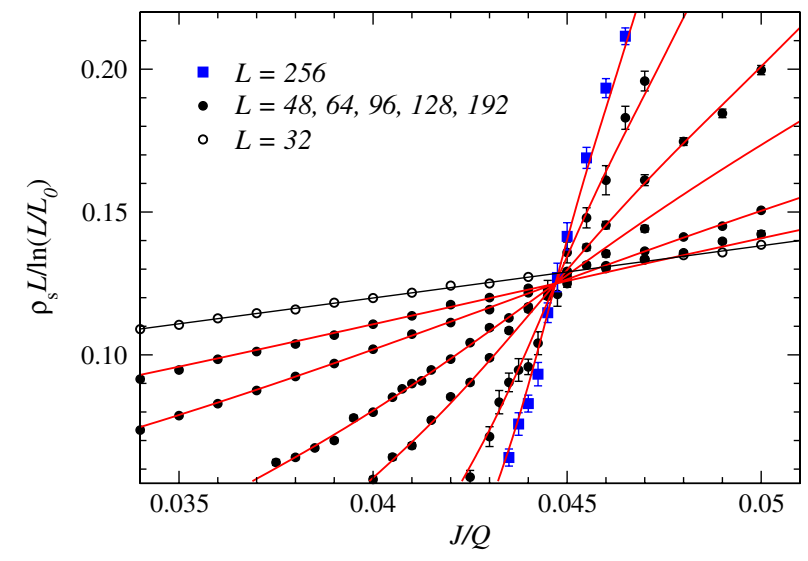

FIG. 5 (color online). Scaling of the spin stiffness with a log correction $\left(L_{0}=0.9\right)$ for $\beta=L$ systems. The curves show a fit to a common polynomial $f\left[\left(J-J_{c}\right) L^{1 / \nu}\right]$ with $J_{c} / Q=0.0447$ and $\nu=0.59$ (not including the $L=32$ data).

systems), along with nonrigorous analytical calculations, has established a consensus that critical points are ubiquitous. The system volumes $\beta L^{2}$ used here for the $J-Q$ model are similar to those in contemporary classical Monte Carlo simulations [27]. In the absence of any concrete signals of first-order behavior, the transition must therefore be regarded as continuous.

The scaling corrections will hopefully stimulate further field-theoretical work to explain them. Scaling anomalies that could be logarithmic have been seen in Monte Carlo studies of the $\mathrm{NCCP}^{1}$ action [3], but it has also been claimed that this action always leads to a first-order transition [19] (in which case a different field theory for the $J-Q$ model would have to be found). Marginal operators leading to logarithms appear in systems at their upper critical dimension, but this is not applicable here. Logarithmic corrections have been previously found in gauge field theories with fermions [28]. On the other hand, conventional power-law corrections due to irrelevant operators are always expected, but here the subleading exponent $\omega$ would have to be very small, which has not been anticipated (although the dangerously irrelevant operator causing the VBS has a small scaling dimension [15] and is a potential source of a small $\omega$ ). Studies of the $\mathrm{SU}(N)$ generalization of the $J-Q$ model would be useful to determine whether $N=2$ is a special case. QMC calculations have already been carried out for $N=3$ and 4 [15], but the quantities discussed here have not yet been investigated.

A consequence of the findings presented here is that the anomalous VBS transition in U(1) symmetric systems [7] should be reevaluated. Scaling deviations very similar to (but stronger than) those in the $J-Q$ model were found, which in $[18,19]$ was interpreted as a first-order transition. Considering scaling corrections, this class of models as well may in the end have continuous transitions [6].

I would like to thank L. Balents, R. Kaul, V. Kotov, R. Melko, F. Nogueira, S. Sachdev, and T. Senthil for useful discussions. This work is supported by NSF Grant No. DMR-0803510.

[1] N. Read and S. Sachdev, Phys. Rev. Lett. 62, 1694 (1989).

[2] T. Senthil, A. Vishwanath, L. Balents, S. Sachdev, and M. P. A. Fisher, Science 303, 1490 (2004).

[3] O. I. Motrunich and A. Vishwanath, Phys. Rev. B 70, 075104 (2004); arXiv:0805.1494.

[4] S. Sachdev and X. Yin, Ann. Phys. (N.Y.) 325, 2 (2010).

[5] A. W. Sandvik, Phys. Rev. Lett. 98, 227202 (2007).

[6] A. W. Sandvik, S. Daul, R. R. P. Singh, and D. J. Scalapino, Phys. Rev. Lett. 89, 247201 (2002).

[7] A. W. Sandvik and R. G. Melko, Ann. Phys. (N.Y.) 321, 1651 (2006)

[8] S. Liang, B. Doucot, and P. W. Anderson, Phys. Rev. Lett. 61, 365 (1988).

[9] P. Henelius and A. W. Sandvik, Phys. Rev. B 62, 1102 (2000).

[10] E. Dagotto and A. Moreo, Phys. Rev. Lett. 63, 2148 (1989); H. J. Schulz, T. Ziman, and D. Poilblanc, J. Phys. I 6, 675 (1996).

[11] O. P. Sushkov, J. Oitmaa, and Z. Weihong, Phys. Rev. B 63, 104420 (2001).

[12] H. G. Evertz, Adv. Phys. 52, 1 (2003).

[13] S. R. White, Phys. Rev. Lett. 69, 2863 (1992).

[14] Analytical many-body techniques [V. N. Kotov, D. X. Yao, A. H. Castro Neto, and D. K. Campbell, Phys. Rev. B 80, 174403 (2009)] and approximate numerical approaches [L. Isaev, G. Ortiz, and J. Dukelsky, J. Phys. Condens. Matter 22, 016006 (2010)] have so far had only limited success with the $J-Q$ model.

[15] J. Lou, A. W. Sandvik, and N. Kawashima, Phys. Rev. B 80, 180414(R) (2009).

[16] R. G. Melko and R. K. Kaul, Phys. Rev. Lett. 100, 017203 (2008); R. K. Kaul and R. G. Melko, Phys. Rev. B 78, 014417 (2008).

[17] F.-J. Jiang, M. Nyfeler, S. Chandrasekharan, and U.-J. Wiese, J. Stat. Mech. (2008) P02009.

[18] A. B. Kuklov, N. V. Prokof'ev, B. V. Svistunov, and M. Troyer, Ann. Phys. (N.Y.) 321, 1602 (2006).

[19] A. B. Kuklov, M. Matsumoto, N. V. Prokof'ev, B. V. Svistunov, and M. Troyer, Phys. Rev. Lett. 101, 050405 (2008).

[20] A. W. Sandvik, Phys. Rev. B 59, R14 157 (1999).

[21] In [16] a "directed loop" SSE method was used, whereas here a straightforward generalization of "deterministic loops" [20] are used.

[22] F. S. Nogueira, Phys. Rev. B 77, 195101 (2008).

[23] M. A. Metlitski, M. Hermele, T. Senthil, and M.P. A. Fisher, Phys. Rev. B 78, 214418 (2008).

[24] K. Binder, Phys. Rev. Lett. 47, 693 (1981); K. Binder and D. P. Landau, Phys. Rev. B 30, 1477 (1984).

[25] K. S. D. Beach, F. Alet, M. Mambrini, and S. Capponi, Phys. Rev. B 80, 184401 (2009).

[26] L. Wang, K. S. D. Beach, and A. W. Sandvik, Phys. Rev. B 73, 014431 (2006).

[27] M. Campostrini, M. Hasenbusch, A. Pelissetto, and E. Vicari, Phys. Rev. B 74, 144506 (2006).

[28] D. H. Kim, P. A. Lee, and X.-G. Wen, Phys. Rev. Lett. 79, 2109 (1997). 